\title{
Acoustic Assessment of an End Mill for Analysis of Tool Wear
}

\author{
Abdullah M. Alzahrani ${ }^{1}$, Rui Liu $^{2}$, and Jason R. Kolodziej ${ }^{3}$ \\ 1,2,3 Rochester Institute of Technology, Rochester, NY 14623, USA \\ aa8247@rit.edu \\ rleme@ rit.edu \\ jrkeme@rit.edu
}

\begin{abstract}
This paper investigates and analyzes the wear on an end mill cutting tool. Current tool condition monitoring systems lack efficient and effective fault prediction and diagnosis. To achieve adoption in the machining industry, condition monitoring must be accurate, non-invasive and cost effective with a longer operational lifetime of tool wear desired. The concept in this work is to associate an acoustic signature to a tool wear condition. First, the application of a Short Time Fourier Transform (STFT) as a time-frequency method to the acoustic measurement that can provide more information throughout the milling operation and gives a better representation of the signal than the conventional methods. Then a support vector machine (SVM) technique is applied as a classification approach to improve condition monitoring of the tool wear. This preliminary research's goal is to create an accurate prognostic tool to determine tool wear condition through a noninvasive means with the ability to retrofit existing mills with the developed technology. The methodology is validated by actual end mill operation of the cutting of an aluminum workpiece at various feed rates, spindle speeds, and cutting depth.
\end{abstract}

\section{INTRODUCTION}

The utilization of sensor based systems for tool conditions monitoring has increased over the past few years and are satisfying the more stringent regulations regarding minimizing maintenance costs, and production losses. Several approaches have been presented to achieve tool condition monitoring and a number of these have been successfully applied in industry. Monitoring of manufacturing processes, development of the intelligent sensors, and decisionmaking abilities are required to fully guarantee high performance (Radhakrishnan \& Kamarthi, 2016). Thus, the development of machine condition monitoring techniques, in the manufacturing environment, are of critical importance to

Abdullah Alzahrani et al. This is an open-access article distributed under the terms of the Creative Commons Attribution 3.0 United States License, which permits unrestricted use, distribution, and reproduction in any medium, provided the original author and source are credited. produce quality products and reduce production downtime. Within this framework, a proper technique of signal processing and understanding machine learning is needed, taking into account the influence of noises on analyzing the cutting process. Furthermore, tool failure during the industrial operation is quite expensive due to the high cost of tools and the time needed to replace

For this work tool wear is the focus and is characterized by the gradual erosion of the cutting tool's surface that is caused by assorted thermo-mechanical mechanisms. Excessive tool wear results in increased tool interface temperatures, ineffective flake origin and flow, poor machining surface finishing quality, and limitation on tool life. It has been known that

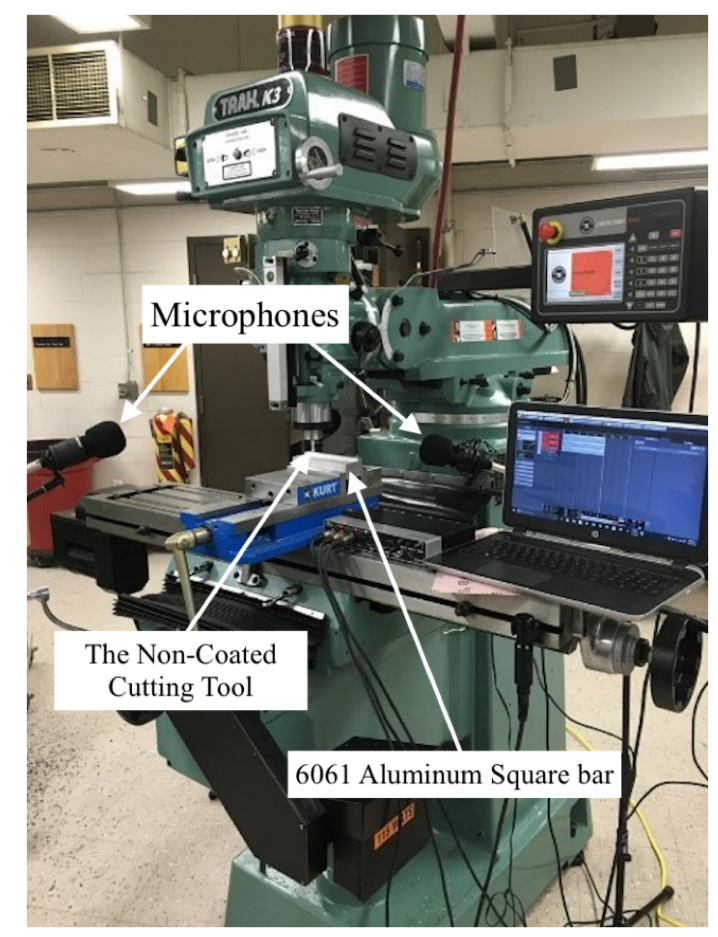

Figure 1. Experimental setup for the TRACK K3 EMX end mill condition monitoring system 
crater wear, flank wear, built-up edge, chipping and breakage are the fundamental process of tool wear (Byrne et al., 1995). It is difficult to measure the tool wear during the cutting operation but vibration and acoustic emission signals have proven to be successful noninvasive methods (Henriquez, Alonso, Ferrer, \& Travieso, 2014) and (Dimla, 2000).

Fundamentally, using indirect sensing is easier than direct, although not as reliable due to outside influences but have the advantage of not influencing the cutting process. Moreover, the condition monitoring hardware can be retrofitted on existing industrial machinery. These techniques utilize different types of sensors: accelerometers, dynamo-meters, current sensors, acoustic emission (AE), etc. sensors. Cutting forces, measured by a dynamo-meter, which is the most effective process variable can be utilized to monitor tool conditions. In this technique, tool wear is observed as the force increase because of a growing friction between the tool and the workpiece. Vibration is another method, because during machining the magnitude grows due to the gradual increase in flank wear. Application of this approach primarily involves the propagation of surface roughness (Dimla, 2002) and (Milfelner, Cus, \& Balic, 2005). The most important drawback of the vibration-based approach is that it is sensitive to the machine speed range and sensor position.

Acoustic measurements are a powerful tool for the study of materials and are especially useful due to their sensitivity to small changes within the material and in-service information. Nonetheless, the technique has not been widely used in the study of tool condition monitoring. In this paper, an experimental application of the acoustic method for identifying tool condition during cutting operations is presented. In the first part, the experimental setup is described including machining conditions and data acquisition. The approach is based on seeded fault testing of the milling of a piece of 6061 aluminum with cutting tools of various levels of degradation using a common end mill. Acoustic measurements are collected and processed in the frequency domain using various spindle speeds and feed rates. Finally, a support vector machine (SVM) classification methodology is implemented to determine the state-of-health of the cutting tool. A summary and future work concludes the paper.

\section{Experimental Setup}

To guarantee that the condition monitoring system can effectively predict the tool wear the cutting conditions have been chosen over an extensive range of cutting speeds and feed rates of the milling machine. The slot milling experiments were conducted on a TRACK K3 EMX vertical end mill (Fig. 1) with high speed numerical control. Five spindle speeds at three feed rates have been chosen for each tool condition. A non-coated, two-flute, high speed steel end mill tool was used for the cutting tool in a vertical milling machine.

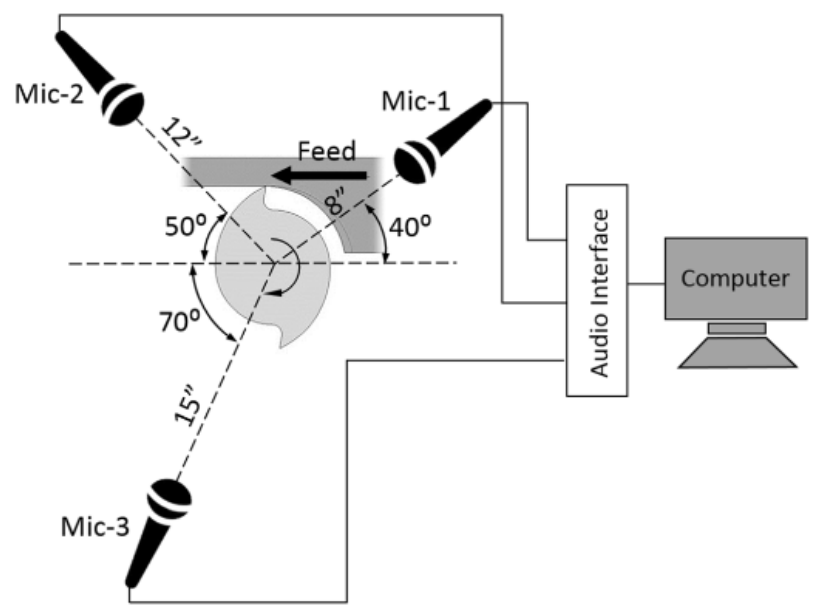

Figure 2. Instrumentation diagram illustrating microphone locations and data acquisition

Three microphones are positioned at 8,12 and 15 inches and angle at 40, 50 and 70 degrees respectively. The complete instrumentation setup is shown in Fig. 2. These microphones were connected to a multi-channel audio system (Steinberg UR44), which were then linked to a computer data acquisition systems. Figure 1 shows the complete end mill experiment system.

The workpiece used in this study is a 6061 Aluminum bar. The machined workpiece is square with a thickness of 0.25 inches and a side length 6 -inches long. The experimental process is shown schematically in Fig. 3. The cutting parameters in this study were constant throughout the cutting process with a radial width of the cutting tool of 0.1875 inches and an axle depth of 0.2 inches. For each tool condition class (Good, Light, Average, Advanced 1, Advanced 2, Failure), five spindle speeds were chosen at three different feed rates resulting in 90 unique cycles of cutting. Noting three microphones are sampling for each cycle results in a total of 270 acoustic data sets. The radial and axial depth of cut have been kept constant during all of the experiments.

This research classifies the six cutting tool wear conditions, with respect to different thickness ranges of flank wear lands on the cutting edges of the tool. The thickness has been computed by averaging various measurements within the cutting zone from the cutting edge tool corner to the distance of axial depth of the cut on the cutting edge. During the slot milling test, the flank wear classification of the cutting tools has been observed periodically with an optical microscope, as shown in Fig. 4. The identification of each tool condition's class as per the level of average flank wear has been defined in Tab. 1 .

All acoustic measurements are collected at a $44.1 \mathrm{kHz}$ sampling rate which is common across audio recording platforms. Table 2 shows a complete list of the seeded fault tests run for 


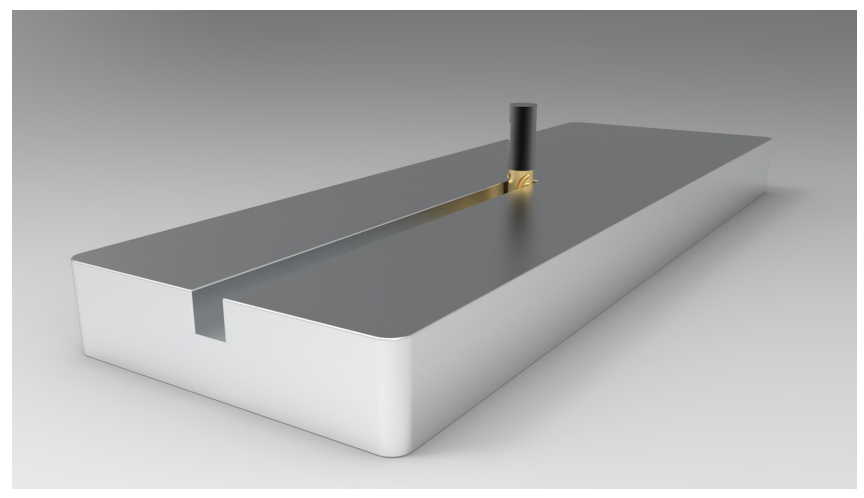

Figure 3. Diagram of end milling experimental setup on 6061 Aluminum workpiece.

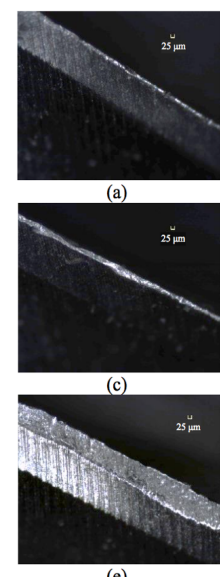

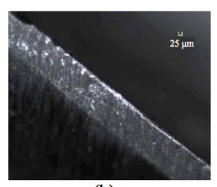
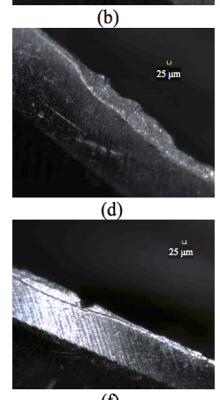

(f)
Figure 4. All types all tool conditions in an optical microscope: (a) Good (b) Light (c) Average (d) Adv. 1 (e) Adv. 2 (f) Failure

Table 1. Tool Wear Classification Levels

\begin{tabular}{|l|l||l|}
\hline No. & Wear Class & THICKNESS RANGE $(\mu \mathbf{m})$ \\
\hline \hline 1 & Good & 0 to 20 \\
\hline 2 & Light & 20 to 40 \\
\hline 3 & Average & 40 to 70 \\
\hline 4 & Advanced 1 & 70 to 100 \\
\hline 5 & Advanced 2 & 100 to 150 \\
\hline 6 & Failure & $>150$ \\
\hline
\end{tabular}

this work. Each cutting tool wear condition is run at five different spindle speeds with each spindle speed having three different feed rates. This results in 90 unique data sets. It is important to note that feed rate and spindle speed was not consistent from wear condition to wear condition. The intent with this approach is to make the classification method accurate irrespective of machine speed.

Figure 5 shows a sample of one of the milling runs. The top
Table 2. Experimental Data with Tool Condition, Spindle Speed, and Feed Rate.

\begin{tabular}{|l|cl|}
\hline Tool Condition & Spindle Feed (RPM) & $\begin{array}{l}\text { Feed } \\
\text { (IPM) }\end{array}$ \\
\hline \hline Good & 1800 & $9,12,13$ \\
Good & 2000 & $10,15,17.5$ \\
Good & 2200 & $12,18,21$ \\
Good & 2300 & $14,20,30$ \\
Good & 2400 & $15,25,40$ \\
\hline Light & 1700 & $8,17,19$ \\
Light & 1800 & $9,16,18$ \\
Light & 1900 & $10,21,22$ \\
Light & 2000 & $12,23,28$ \\
Light & 2200 & $14,26,35$ \\
\hline Average & 1500 & $7,13,14$ \\
Average & 1700 & $8,15,16$ \\
Average & 1900 & $9,19,20$ \\
Average & 2100 & $10,21,24$ \\
Average & 2300 & $11,21,33$ \\
\hline Advanced 1 & 1400 & $6.5,12,14$ \\
Advanced 1 & 1600 & $7,13,15$ \\
Advanced 1 & 1800 & $7.5,16,17$ \\
Advanced 1 & 2000 & $8,18,20$ \\
Advanced 1 & 2200 & $9,22,25$ \\
\hline Advanced 2 & 1300 & $5.5,9,10$ \\
Advanced 2 & 1500 & $6,13,15$ \\
Advanced 2 & 1600 & $6.5,14,17$ \\
Advanced 2 & 1800 & $7,18,20$ \\
Advanced 2 & 2100 & $8,22,25$ \\
\hline Failure & 1200 & $5,10,13$ \\
Failure & 1400 & $6,11,12$ \\
Failure & 1500 & $7,14,15$ \\
Failure & 1800 & $8,18,20$ \\
Failure & 2000 & $9,22,24$ \\
\hline & & \\
\hline
\end{tabular}
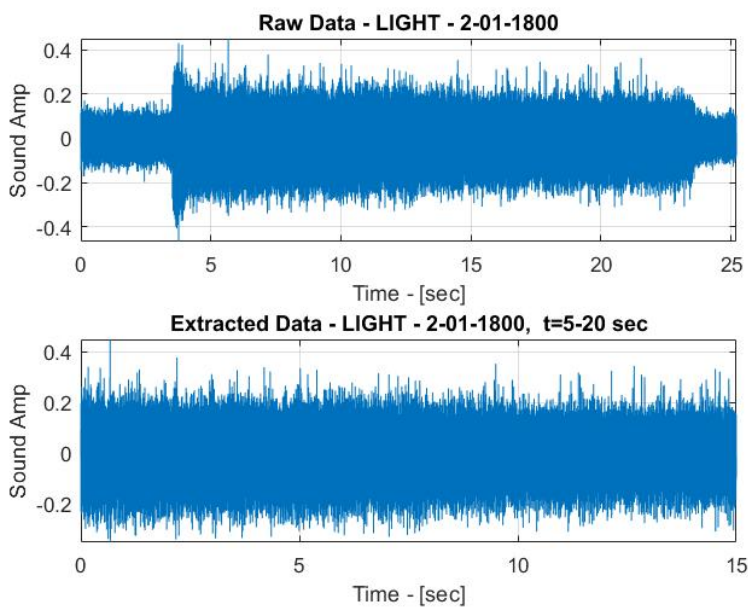

Figure 5. Raw acoustic measurement for tool condition $=$ light, spindle speed $=1800$, feed rate $=2$, microphone $=1$ (top). Extracted data set $(\mathrm{t}=5-20)$ minus startup and shutdown events.

plot shows the complete run including start up and shutdown. From the time the tool makes contact with the workpiece to the time the tool leaves the workpiece the material type is homogeneous and thus the acoustic measurement can be as- 


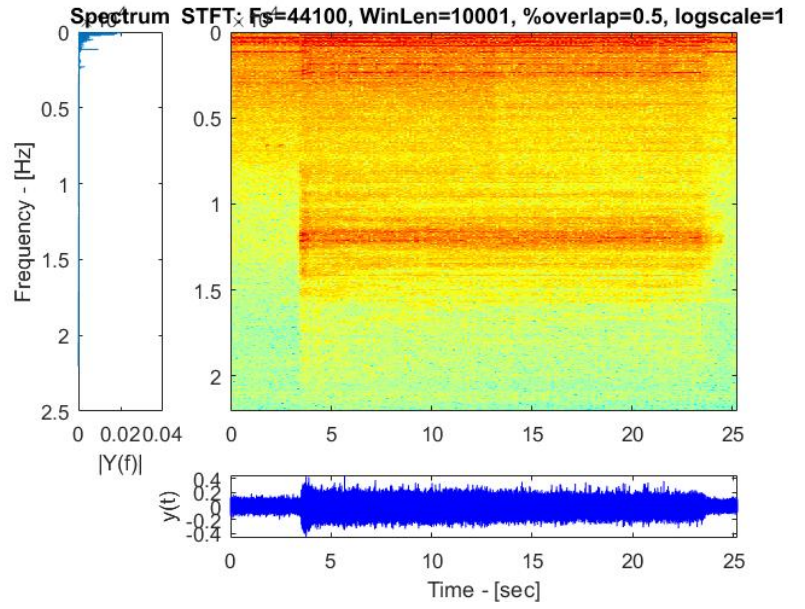

Figure 6. STFT representation of full cutting cycle for the Light tool wear condition at 1800RPM spindle speed at feed rate \#2 from microphone \#1.

sumed a stationary process. The bottom plot in the figure is the extracted data set for the entire cutting process minus the transient events.

\section{Signal Processing \& Classification}

To ensure that the process can be assumed stationary a shortterm Fourier transform (STFT) is determined and the result analyzed visually to observe the frequency spectrum changes throughout the cutting cycle. Figure 6 shows the STFT of the complete cutting cycle including the startup and shutdown transients. From this plot it is reasonably safe to assume that the frequency character does not chance sufficiently while the cutting tool is engaged with the workpiece as evidence by the constant frequency bands shown throughout the cut.

Next, the extracted data set is segmented into one second samples and converted to the frequency domain by fast Fourier transform (FFT). A zoomed example of this spectrum is shown in Fig. 7, where the top figure shows the spectrum between $0-1000 \mathrm{~Hz}$, which is a significant reduction of the complete spectrum which continues to $22,050 \mathrm{~Hz}$. The bottom figure further zooms the spectrum to a range of $0-200 \mathrm{~Hz}$. The intent of both of these figures is to illustrate that the three microphones at various angles and distances identify nearly the exact sample frequency peaks as one would expect. The magnitudes would have variation due to distance from the work piece and amplifier gain settings but the identified frequency content of the signals appears to be very repeatable. Note that for the proposed method the full frequency spectrum up to the Nyquist sampling limit is used in this classification method described later. The zoomed plots shown in Fig. 7 are simple shown to show repeatability of the sensing method.

Each data file comprising of a single cutting operation is nom-

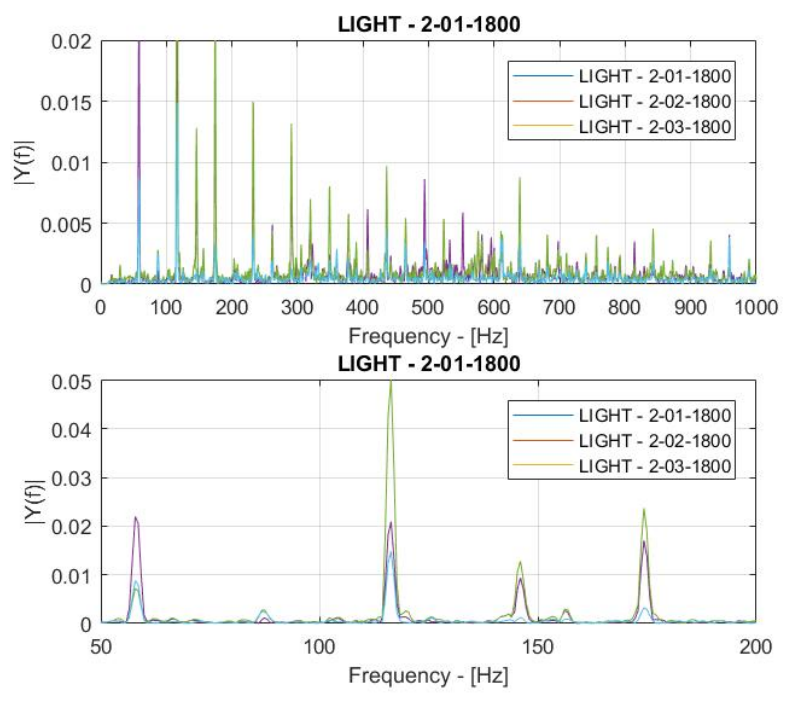

Figure 7. FFT representation of a 1-second extracted data sample of a cutting cycle for the Light tool wear condition at 1800RPM spindle speed at feed rate of \#2 from microphones $\# 1$, \#2, and \#3. From $0-1000 \mathrm{~Hz}$ (top) and zoomed from 0 $200 \mathrm{~Hz}$ (bottom)

inally 20-30 seconds long depending on the feed rate. With the initial workpiece engagement and departure events removed, this results in approximately 20 one second data segments for a given cutting tool wear, spindle speed, and feed rate. All three microphones record every test. Each one second, 44,100 sample, acoustic measurement is converted to the frequency domain by FFT. To further show that throughout the cutting operation the acoustic signature is indeed a stationary process, Figure 8 is a waterfall plot showing 30 frequency spectra from consecutive data samples during the cutting of a workpiece with Advance 1 level of wear. From the figure it is clear that the $0-500 \mathrm{~Hz}$ spectra are visually very similar. For this preliminary work the entire magnitude frequency spectrum is used as a feature vector to the classification scheme. Since each data file is of the same length, the length of the feature vector is consistent. Thus, at a point-bypoint basis the elements of the vector can be compared. The tool wear health prediction classification method applied in this work is a Support Vector Machine (SVM).

\subsection{SVM Technique}

SVMs are an advanced computational learning technique which is based on statistical learning theory and have been utilized effectively in many real world classification problems like image classification, Bioinformatics classification, and hand written character recognition (Widodo \& Yang, 2007). The primary benefit of SVM classification and the reason it is employed in this work is because it is designed to be very effective in dealing with large datasets where the dimension of feature vectors does not impact the performance of the SVM. 
This allows for selecting more statistical features in the case of fault diagnosis. As mentioned above the actual magnitudes of the frequency spectrum at specific, and consistent, frequencies are used in the actual feature vector for each given acoustic data sample. This result in a comparatively very large feature vector but as will be shown still meets with a high degree of tool wear classification accuracy when applied using a trained SVM.

The following is a very brief outline of the SVM technique, but the interested reader is encouraged to investigate any of the numerous texts on the subject such as (Cristianini \& Shawe-Taylor, 2000).

Taking the input data to be $X_{k}$ where $k=[1,2,3, \ldots, N], N$ represents the total number of samples. The samples of this data set $X_{k}$ to have two classes namely negative and positive classes. Each class is assigned class labels, $y_{k}=[1,-1]$, and represents the positive and negative class, respectively. A hyperplane which obtains the class boundaries $G(x)=0$, from which the linear classifier can be written as

$$
G(x)=w^{T} x+b=\sum_{k=1}^{N} w_{k} x_{k}+b=0,
$$

where $w$ is $N$-dimensional vector and $b$ is scalar. The vector $w$ and scalar $b$ are utilized to obtain the location of the separating hyperplane. The main goal of this approach is to determine a hyperplane which maximizes the distance between the plane and the nearest data point, it is called optimal hyperplane. This requires determining $w$ and $b$ while maximizing $N=\frac{1}{\|w\|}$. A adjusted optimization problem with quadratic objective and linear constraints is given by,

$$
\min _{w, b} \frac{1}{2}\|w\|^{2}
$$

subject to,

$$
y_{k} G\left(x_{k}\right)=y_{k}\left(w^{T} x_{k}+b\right) \geq 1, \quad \forall k=1,2, \ldots, N .
$$

For this work 90 different conditions were tested for the six levels of tool wear as outlined in Tab. 2. During the test the three microphones recorded approximately 30 seconds of acoustic data per test. After removing the startup and shutdown part of the audio signal the sound file is segmented into one second sound bytes. These sound bytes are then transformed to the frequency domain as described previously. Randomly chosen, $70 \%$ of the one second files are used to train the SVM classifier with $30 \%$ retained to be used as a validation data set. The magnitude from the entire frequency spectrum of a one second acoustic signal is used as the feature vector. The feature vectors for all wear conditions, independent of spindle speed and feed rate, is compiled into the

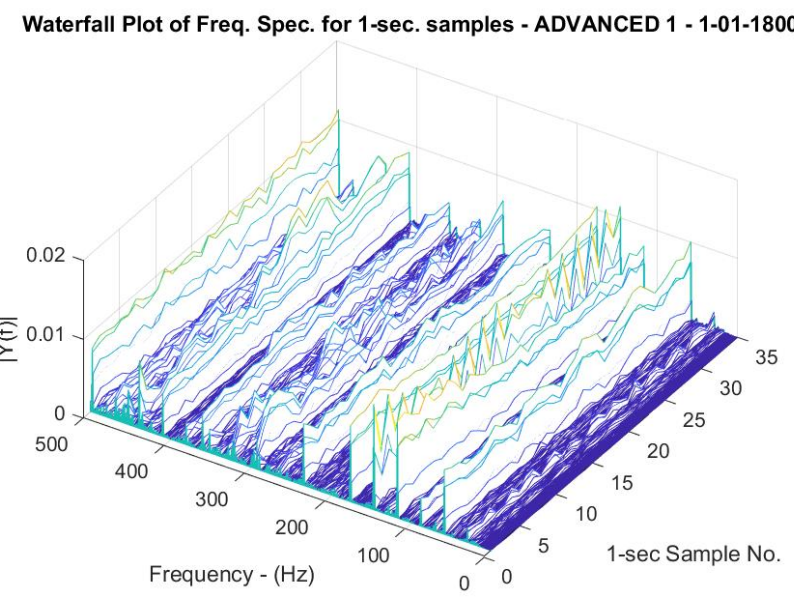

Figure 8. Waterfall plot all 1-second extracted data samples of a cutting cycle for the Advanced 1 tool wear condition at 1800RPM spindle speed at feed rate of \#1 from microphones $\# 1$, from $0-500 \mathrm{~Hz}$.

complete input set used to train SVM. The only output is the known wear condition. The intent of this approach is to accurately classify the heath of the cutting tool regardless of the settings of the end mill. The SVM only has knowledge of the frequency spectrum. Once the SVM is trained the validation set of sound signals is sent through the classifier with the results shown as a confusion matrix in Fig. 9 with a table of prediction accuracy in Tab. 3. It is clear that the trained SVM is able to identify the level of tool wear to a very high degree.

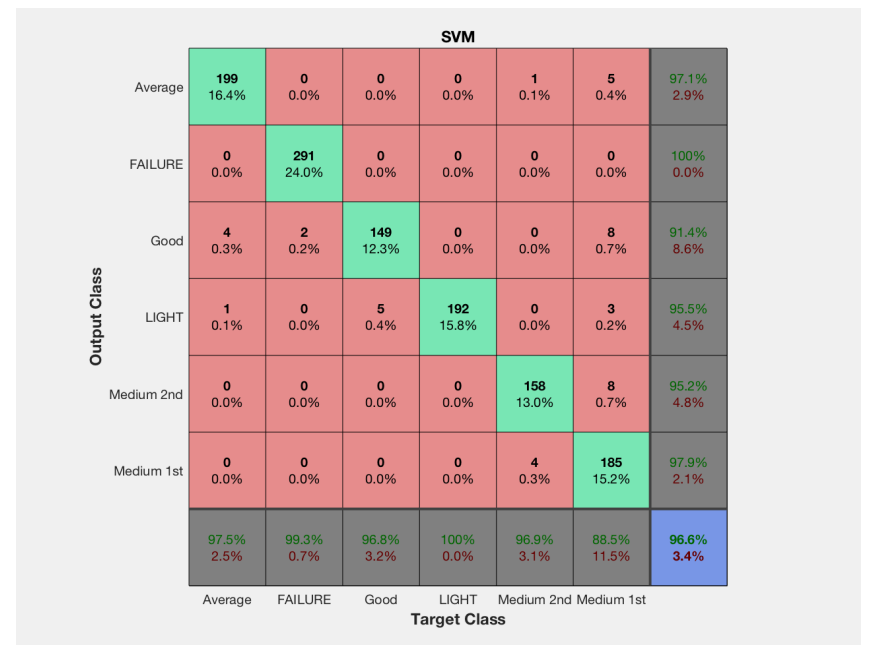

Figure 9. Comparison of tool wear class prediction with actual wear class.

\subsection{Histogram Features}

To compare the success of the proposed method a simple histogram approach is presented. Intentionally, the spindle speed and feed rate was not kept common during the testing, but 
Table 3. Performance analysis of the algorithm for each class.

\begin{tabular}{|l|l|l|l|}
\hline NO. & Wear Class & Precision (\%) & Error $(\%)$ \\
\hline \hline 1 & Good & 91.40 & 8.60 \\
\hline 2 & Light & 95.50 & 4.50 \\
\hline 3 & Average & 97.10 & 2.90 \\
\hline 4 & Adv.1 & 97.90 & 2.10 \\
\hline 5 & Adv.2 & 95.20 & 4.80 \\
\hline 6 & Failure & 100.00 & 0.00 \\
\hline
\end{tabular}

there are a few conditions with overlapping conditions. For a spindle speed of 1800 RPM and a feed rate of 18 IPM there were three unique tool wear conditions: Light, Advanced 2, and Failure. The objective of this simple test is to determine if using sound magnitude alone could be used to classify the tool condition. Again using the raw audio signal with the startup and shutdown removed the remaining acoustic measurement is broken into 0.1 second bytes (441 points) and a signal RMS calculated. This collection of RMS values for a given tool wear condition is then plotted as a histogram. The three wear conditions for which spindle speed and feed rate are common are shown in Fig. 10. While there is indeed some separation between the classes it is clear that the likelihood of accurate classification would not exceed the proposed method using the SVM.

\section{Conclusion}

This work presents preliminary research into developing a tool wear condition monitoring method using non-invasive acoustic measurements. A common machine shop end mill has been setup with three precision microphones at various distance from the cutting process. Six cutting tools of increasing levels of degradation are use to mill a slot in a piece of aluminum at constant spindle speeds and feed rates. The acoustic signals are broken into many one second sound bytes and processed with a traditional FFT technique. The full

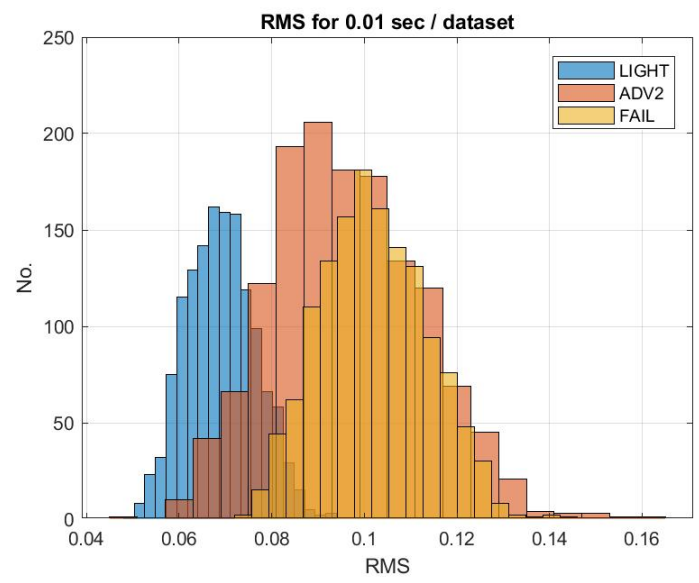

Figure 10. Histogram of the three tool ware conditions (Light, Advanced 2, and Failure) for a 0.01 second sound segment from the middle of the cutting operation. magnitude spectrum is then used to train a SVM classification technique. It is shown through validation data that the method is highly successful at classifying the level of tool wear. Due to the preliminary nature of this work the amount of future work planned is considerable at present mostly in investigating the parameter space. For example, determining the effect of the size of the aluminum bar, or if the cutting tool is inserted into the mill slightly different depth, does the method work on different work piece material (i.e. steels?) More theoretical work is planned as well, such as looking at the frequency spectrum and relating only key portions of it and how it relates to the workpiece material and geometry. Finally, additional work is planned to develop a method to identify work piece hardness variations.

\section{REFERENCES}

Byrne, G., Dornfeld, D., Inasaki, I., Ketteler, G., Konig, W., \& Teti, R. (1995). Tool condition monitoring: the status of research and industrial application. Annals of the CIRP, 44(2), 541-567.

Cristianini, N., \& Shawe-Taylor, J. (2000). An introduction to support vector machines and other kernel-based learning methods. Cambridge University Press.

Dimla, D. E. (2000). Sensor signals for tool-wear monitoring in metal cutting operations - a review of methods. International Journal of Machine Tools and Manufacture, 40(8), 1073 - 1098.

Dimla, D. E. (2002). The correlation of vibration signal features to cutting tool wear in a metal turning operation. The International Journal of Advanced Manufacturing Technology, 19(10), 705-713.

Henriquez, P., Alonso, J., Ferrer, M., \& Travieso, C. (2014). Review of automatic fault diagnosis systems using audio and vibration signals. IEEE Transactions on Systems, Man, and Cybernetics: Systems, 44(5), 642-652.

Milfelner, M., Cus, F., \& Balic, J. (2005). An overview of data acquisition system for cutting force measuring and optimization in milling. Journal of Materials Processing Technology, 164-165, 1281 - 1288.

Radhakrishnan, S., \& Kamarthi, S. (2016). Complexityentropy feature plane for gear fault detection. In 2016 ieee international conference on big data (p. 20572061).

Widodo, A., \& Yang, B.-S. (2007). Support vector machine in machine condition monitoring and fault diagnosis. $\mathrm{Me}$ chanical systems and signal processing, 21(6), 25602574. 


\section{BIOGRAPHIES}

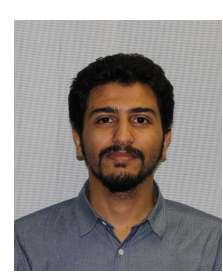

Abdullah Alzahrani is a Ph.D. student in the Electrical Engineering department at the Rochester Institute of Technology (RIT) in Rochester, NY. He received his B.S. in electrical engineering in 2014 from Northern Border University located in Arar, KSA and in 2018 his M.S. in electrical engineering from RIT. His research interests are in the application of optimization techniques and policy analysis to electrical power and control system. Member at Saudi Council of Engineering.

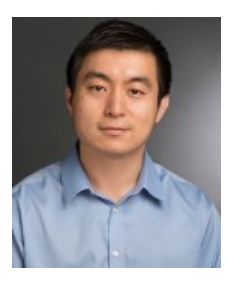

Rui Liu is an Assistant Professor of Mechanical Engineering at the Rochester Institute of Technology (RIT) in Rochester, NY. He received his B.Sc. degree in Jet Propulsion from Beijing University of Aeronautics \& Astronautics in 2005, the M.Sc. degree in Mechanical Engineering at Northeastern University in 2010, and the Ph.D. degree in Mechanical Engineering at Georgia Institute of Technology in 2014. His research interests include machining simulation and cutting tool condition monitoring. He is an active member of ASME, SME, and AGMA.

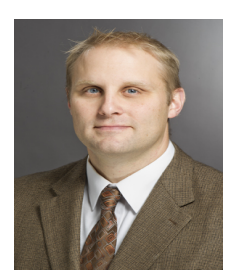

Jason R. Kolodziej is an Associate Professor of Mechanical Engineering at the Rochester Institute of Technology (RIT) in Rochester, NY. He received his Ph.D. in mechanical engineering from the State University of New York at Buffalo in 2001 with a research focus in controls and nonlinear system identification. For eight years he worked in industry for General Motors Fuel Cell Activities as a Sr. Research Engineer with principle duties in hybrid electric-fuel cell vehicle powertrain controls and system architecture. To date he has been granted 10 U.S. Patents. His present research focus is the study of fault detection, diagnosis, and prognostic health assessment of engineering systems. He currently has funded projects covering a wide range of industrial applications from: electromechanical actuators in aircrafts to fuel cell automotive powertrains to large scale compression equipment. He is a member of the ASME. In 2012, he was awarded RITs prestigious Eisenhart Provost Award for Excellence in Teaching. 